\title{
Morphogen profiles can be optimized to buffer against noise
}

\author{
Timothy E. Saunders and Martin Howard \\ Department of Computational and Systems Biology, John Innes Centre, Norwich NR4 7UH, United Kingdom \\ (Received 13 March 2009; revised manuscript received 26 August 2009; published 2 October 2009)
}

\begin{abstract}
Morphogen profiles play a vital role in biology by specifying position in embryonic development. However, the factors that influence the shape of a morphogen profile remain poorly understood. Since morphogens should provide precise positional information, one significant factor is the robustness of the profile to noise. We compare three experimentally relevant classes of morphogen profiles (linear, exponential, and algebraic) to see which is most precise when subject to both external embryo-to-embryo fluctuations and internal fluctuations due to intrinsically random processes such as diffusion. We find that both the kinetic parameters and the overall gradient shape (e.g., exponential versus algebraic) can be optimized to generate maximally precise positional information.
\end{abstract}

DOI: 10.1103/PhysRevE.80.041902

PACS number(s): 87.18.Tt, 87.10.-e, 87.17.Pq

\section{INTRODUCTION}

Morphogens are signaling molecules which play a vital role in biological development by inducing responses in a concentration-dependent manner [1]. In the standard model of morphogen gradients, morphogen proteins originate from a localized source, diffuse and are degraded, setting up a concentration gradient across the system. This gradient can control patterns of gene expression, where, for example, a gene is switched on when the concentration is above a certain fixed threshold, but is off otherwise. In this work, we focus on morphogen gradients specifying boundaries of gene expression at fixed absolute distances from the morphogen source, a scenario that is frequently realized in developmental biology.

Developmental systems need to be robust to sources of noise in order to generate precise patterns of gene expression. Here, we address a simple question: in the presence of noise, which morphogen profile is most precise in specifying the positions of gene expression boundaries? In principle, any spatially nonuniform profile could be used to position gene expression boundaries; our goal is to understand which profiles might be preferred. Previous theoretical approaches have predominantly examined robustness of morphogen profiles to embryo-to-embryo fluctuations in the morphogen production rate [2-4] and analysis suggests that algebraic profiles are most precise [2]. However, even in (hypothetical) embryos with no embryo-to-embryo fluctuations, there would still be variation in positional information due to internal fluctuations in morphogen production, diffusion, and degradation. Such fluctuations impose limits on the precision of biochemical signaling [5-7] and could in principle alter the shape of the profile with the best precision. Internal fluctuations are particularly large if the morphogen is, directly or indirectly, a transcription factor. In that case, the arrival of a morphogen molecule at the nanometer-scale DNA binding sites on the target genes will be a rare stochastic event $[7,8]$. Moreover, internal fluctuations in the morphogen production rate may also play an important role [9].

In the classical picture of morphogen gradients, as presented above, morphogens provide positional information independent of other processes within the embryo. However, in real systems, boundary specification is more complex than this, with multiple control mechanisms involved. A standard example is the Bicoid (Bcd) gap-gene network in the fruit fly Drosophila melanogaster [10]. Bcd is a morphogen that promotes the expression of target genes such as hunchback $(h b)$. A network of gap genes then further influences precise $h b$ boundary positioning [10]. Despite the apparent complexity of such systems, spatial positioning is still often determined primarily by a morphogen gradient. This has been demonstrated for the Bcd- $h b$ network in Drosophila by analyzing a mutant that has the appropriate gap-gene elements removed. Such a mutant is still able to precisely define the $h b$ expression boundary with an error of only $\sim 2 \%$ embryo length though this is somewhat less precise than wild-type embryos, with error $\sim 1 \%$ embryo length [10]. Furthermore, Bcddriven reporters that are insensitive to gap-gene regulation can also precisely specify boundaries $[11,12]$. In this paper we therefore explore the precision with which a single morphogen gradient can define spatial positions independent of other regulatory factors. From the above experiments, we see that this approach is reasonable as morphogen gradients can provide precise positional information independent of the other regulatory components that act to sharpen domain boundaries.

Recent experiments in Drosophila have quantitatively studied the morphogen proteins Bcd [8,13,14], Decapentaplegic (Dpp) [15], and Wingless [15]. Interestingly, the observed profiles are exponentially decaying in all cases. In order to better understand this finding, we focus on three classes of experimentally relevant morphogen profiles (linear, exponential, and algebraic) to see which is most precise when subjected to the combined effects of both external and internal fluctuations. We find that the kinetic parameters describing morphogen profiles can be optimized to buffer against the combined effects of both sources of noise. By comparing optimized profiles, we then see that the overall shape of the profile (e.g., exponential versus algebraic) can also be optimized. Of the three profiles, exponential profiles frequently emerge as the best compromise: such profiles are not particularly robust to either external or internal fluctuations taken singly, but when both types of fluctuation are taken together, exponential profiles can be the most precise. We therefore propose a simple design principle for morpho- 
gen profiles, namely, that evolution has selected gradients with optimal robustness to the combined effects of embryoto-embryo and internal noise. Depending on which source of noise is most important, qualitatively different morphogen profiles will be selected. Given that very high positional precision can be achieved by morphogens (e.g., a few percent of embryo length in the Bcd system), it seems plausible that optimization may well be exploited by evolution. However, there may still be other constraints on morphogen systems, for example, on the information capacity of the signaling network $[16,17]$.

\section{MODELS}

We consider three representative models of morphogen gradients: a freely diffusing morphogen with a source and a sink [18], diffusion with linear decay, and diffusion with quadratic decay [2]. Why study these three particular models? Diffusion with linear decay leads to exponential morphogen profiles that have been observed experimentally. Nonlinear decay mechanisms leading to algebraic profiles are robust to external morphogen production fluctuations [2] and we study quadratic decay (decay via dimerization) as a representative example [2]. Bone morphogenetic protein (BMP) in Xenopus embryos is a possible candidate for a morphogen shaped by such effective nonlinear degradation [19]. Finally, the source-sink model generating a linear profile provides insight into gradients generated by diffusion with localized degradation [18]. This is realizable when the morphogen degradation factors are tightly localized, such as for retinoic acid along the anterio-posterior axis of mice embryos [20].

A three-dimensional system is considered with a planar source of morphogen at $x=0$ that produces a flux of proteins $(J)$ which diffuse $(D)$ through the system (length $L$ ). In the absence of fluctuations the morphogen concentration is given by the reaction-diffusion equation

$$
\partial_{t} \rho(\mathbf{x}, t)=D \nabla^{2} \rho(\mathbf{x}, t)-\psi \rho(\mathbf{x}, t)^{n},
$$

where $\psi=\mu\left[\mathrm{s}^{-1}\right](n=1)$ and $\psi=\alpha\left[\mu \mathrm{m}^{3} \mathrm{~s}^{-1}\right](n=2)$. The boundary conditions are $\left.D \partial_{x} \rho(x)\right|_{x=0}+J=0$ and, for $n=1,2$, $\left.D \partial_{x} \rho(x)\right|_{x=L}=0$. The source-sink model corresponds to $\psi=0$ and a sink at the boundary $x=L[$ i.e., $\rho(L)=0]$. The steadystate solutions to Eq. (1) depend only on the linear distance from the source in the $x$ direction:

$$
\begin{gathered}
\rho_{s-s}(x)=(J / D)(L-x), \\
\rho_{\text {lin }}(x)=(J \lambda / D) e^{-x / \lambda}, \\
\rho_{\text {quad }}(x)=A\left(x+x_{0}\right)^{-2},
\end{gathered}
$$

where $\rho_{s-s, \text { lin,quad }}$ correspond to the source-sink, linear decay, and quadratic decay models, respectively, and $\lambda=\sqrt{D / \mu}, A$ $=6 D / \alpha, x_{0}=\left(12 D^{2} / J \alpha\right)^{1 / 3}$ with Eqs. (3) and (4) valid for $x, x_{0}, \lambda \ll L$.

\section{EXTERNAL FLUCTUATIONS}

We first investigate robustness to external fluctuations solely in $J$ as this is believed to be an experimentally relevant scenario [8]. The position $x_{T}$ where the concentration passes through a threshold $\left(\rho_{T}\right)$ varies as a result of embryo-toembryo fluctuations $(\delta J)$ in the morphogen production rate. Keeping the threshold concentration fixed, and expanding around $x_{T}$ to leading order, the width due to external fluctuations is given by $W \approx \Delta \rho\left(x_{T}\right) /\left|\left\langle\rho^{\prime}\left(x_{T}\right)\right\rangle\right|$. Here, $\Delta \rho\left(x_{T}\right)$ $=\left[\left\langle\rho\left(x_{T}\right)^{2}\right\rangle-\left\langle\rho\left(x_{T}\right)\right\rangle^{2}\right]^{1 / 2}(\langle\ldots\rangle$ denotes ensemble averaging $)$ are the variations due to external embryo-to-embryo fluctuations, and $\rho^{\prime}\left(x_{T}\right)=\left.\partial_{x} \rho\right|_{x=x_{T}}$. The widths for the three models are $W_{s-s} \approx\left(L-x_{T}\right)(\delta J / J), \quad W_{\text {lin }} \approx \lambda(\delta J / J)$, and $W_{\text {quad }}$ $\approx(1 / 3) x_{0}(\delta J / J)$, to leading order in $\delta J / J$. Typically, $x_{T}$ $\sim L / 2$ (as in the Bcd controlled $h b$ gene expression boundary in Drosophila) and $x_{0}<3 \lambda$, leading to $W_{\text {quad }}<W_{\text {lin }}<W_{s-s}$.

\section{INTERNAL FLUCTUATIONS}

Within an embryo internal fluctuations are also an important source of noise. We consider morphogen production, diffusion, and (if appropriate) degradation to be stochastic processes. We make two important assumptions about the sampling of the morphogen gradient. First, we assume that the morphogen concentration is sampled in a region of linear size $\Delta x$ corresponding to the size of the binding site at target genes [8]. Second, we do not explicitly include details of the binding process of the morphogen to the appropriate DNA regulatory region [6]. Incorporating such an effect reduces the sampling effectiveness, thus increasing the effects of internal noise, but does not qualitatively change our results. In effect, we assume that the system is able to perfectly sample the morphogen gradient. Internal fluctuations in particle density within the sampling volume again cause the position where the gradient passes through $\rho_{T}$ to vary leading to imprecision in the positional information provided by the gradient. The width due to internal fluctuations $\omega$ is again given by $\omega \approx \Delta \rho\left(x_{T}\right) /\left|\left\langle\rho^{\prime}\left(x_{T}\right)\right\rangle\right|[7]$, but where $\Delta \rho$ is now due to internal fluctuations. In the source-sink and linear decay models the statistics of the particle number $n(x)$ due to internal fluctuations are Poissonian, since both models are linear and diffusion and degradation (if present) are Poisson processes $[7,21,22]$. We also assume that fluctuations in the morphogen production rate are described by Poisson statistics. It is possible that the effective fluctuations in production are non-Poissonian [23]. However, we have confirmed numerically that our results are dominated by diffusive fluctuations: fluctuations in morphogen production make no difference to our results. We present numerical results below demonstrating that the particle number statistics in the quadratic decay model are also effectively described by a Poisson distribution. This result is not obvious, as nonlinear decay processes could, in principle, generate non-Poisson fluctuations. In all cases, we assume that diffusion of morphogen proteins is a purely three-dimensional process (there could be onedimensional sliding along DNA but this leads to similar fluctuations as three-dimensional diffusion [24]).

\section{SIMULATIONS}

Stochastic simulations were performed on a threedimensional lattice containing discrete particles with particle 

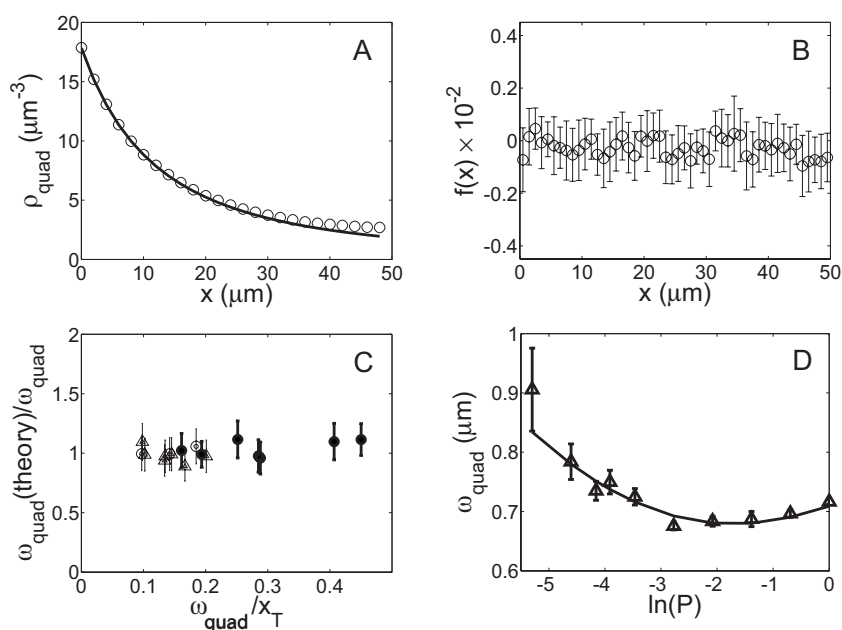

FIG. 1. Numerical results: (a) $\rho_{\text {quad }}$ against $x$ averaged for $\tau$ $=5000 \mathrm{~s}$. Line is fit to Eq. (4). (b) Mean and standard deviation of $f(x)$ (error bars from ten simulations). (c) Comparing $\omega_{\text {quad }}$ from simulations with Eq. (7) for $J=1(\bullet), J=2(\bigcirc)$, and $J=5(\triangle)$ molecules $\mu \mathrm{m}^{-2} \mathrm{~s}^{-1}$, with various $\tau$ and $x_{T}$. (d) $\omega_{\text {quad }}$ against nonlinear decay probability averaged for $\tau=1000 \mathrm{~s}$. Line is prediction from Eq. (7), where $\alpha=P \gamma /(1+c P \gamma) \quad[25] \quad\left[\gamma=(\delta x)^{3} / \delta t, \quad c\right.$ $\left.=12.5 \mu \mathrm{m}^{-3} \mathrm{~s}\right]$. Parameters unless stated otherwise: $J$ $=1$ molecule $\mu \mathrm{m}^{-2} \mathrm{~s}^{-1} ; \quad x_{T}=3 \mu \mathrm{m} ; \quad D=D_{0}=0.67 \mu \mathrm{m}^{2} \mathrm{~s}^{-1} ; \quad P$ $=10^{-2} ; L=50 \mu \mathrm{m}$; lattice spacing $\delta x=0.01 \mu \mathrm{m}$; time step $\delta t=2.5$ $\times 10^{-4} \mathrm{~s}$

injection on the plane $x=0$, diffusion, and (if appropriate) degradation. For the quadratic decay model, simulations were performed using a range of parameter values for $J, D$, and $P$ (probability of two particles degrading within a single time step given that they occupy the same lattice site) and system size $L$. In Fig. 1(a) we demonstrate that concentrations measured in our simulations agree well with Eq. (4) (the finite-size effects at large $x$ do not alter our conclusions). To confirm that particle number fluctuations are described by Poisson statistics for the quadratic decay model we calculated $f(x)=\left[\left\langle n(x)^{2}\right\rangle-\langle n(x)\rangle^{2}-\langle n(x)\rangle\right] /\langle n(x)\rangle$, where each simulation was averaged for $5000 \mathrm{~s}$ [Fig. 1(b)]. In three dimensions diffusion is efficient enough to prevent the buildup of non-Poissonian correlations resulting from the quadratic decay reactions. This result is robust to parameter variations in our simulations (data not shown).

\section{TIME AND SPATIAL AVERAGING}

We now consider the effects of time and spatial averaging $[7,8]$, which act to reduce $\omega$. Time averaging is performed by the downstream signal-processing network, where the time scale is typically given by the lifetimes of the transcripts and/or proteins of the target gene. As discussed above, we assume perfect time averaging of the morphogen concentration. Though this process is noisy, recent analysis of the Bcd signaling pathway suggests that input noise dominates over output noise in expression of the $h b$ target gene supporting our assumption [26]. Over an averaging period $\tau$, there can, at most, be $N_{\tau}=\tau / \tau_{\text {ind }}$ independent readings of the morphogen concentration which reduce the measurement width by a factor $\sim N_{\tau}^{-1 / 2}$. Intuitively, $\tau_{\text {ind }} \sim(\Delta x)^{2} / D_{0}$, the typical time scale for the sampling volume to empty and then be refilled by new protein particles via diffusion $[5,7]\left(D_{0}\right.$ is "local" diffusion responsible for movement into the sampling volume, which may not equal $D$, the bulk diffusion). A constant associated with time averaging is found numerically (see below). We also include spatial averaging, motivated by recent experimental [8] and theoretical work [27] on the Bcd- $h b$ signaling pathway. In particular, results from [27] have demonstrated that diffusion of the target protein $\mathrm{Hb}$ can result in spatial averaging and increased target domain precision. By averaging over a number of different nuclei and/or cells, $N_{\text {spat }}$, the effects of internal fluctuations can be further reduced by a factor $\sim N_{\text {spat }}^{-1 / 2}$. From [8], $N_{\text {spat }} \approx C D_{0} \tau$ where $C$ is a constant which depends on the particular arrangement of nuclei and/or cells. We emphasize that our key results, that morphogen profiles and kinetic parameters can be optimized to buffer against noise, are not dependent on the inclusion of such spatial averaging.

\section{WIDTH DUE TO INTERNAL FLUCTUATIONS}

For the three models we find that the widths $\omega$ due to internal fluctuations are

$$
\begin{gathered}
\omega_{s-s}^{2}=\frac{k_{0}^{2}\left(D / D_{0}\right)}{\tau J \Delta x N_{\text {spat }}}\left(L-x_{T}\right), \\
\omega_{\text {lin }}^{2}=\frac{k_{1}^{2}\left(D / D_{0}\right)}{\tau J \Delta x N_{\text {spat }}} \lambda e^{x_{T} / \lambda}, \\
\omega_{\text {quad }}^{2}=\frac{k_{2}^{2}\left(D / D_{0}\right)}{2 \tau J \Delta x N_{\text {spat }}} \frac{\left(x_{T}+x_{0}\right)^{4}}{x_{0}^{3}} .
\end{gathered}
$$

In Fig. 1(c), we compare Eq. (7) with simulation results for a range of parameter values. We find good agreement between the two approaches, where $k_{2}=0.56 \pm 0.06$ is a fitting parameter. A similar procedure for the other two models yields $k_{0}$ $=0.53 \pm 0.07$ and $k_{1}=0.60 \pm 0.05$.

\section{OPTIMIZING KINETIC PARAMETERS}

Importantly, the underlying kinetic parameters in the linear and quadratic decay models can be optimized to minimize $\omega_{\text {lin }}$ and $\omega_{\text {quad }}$ at the threshold position $x_{T}$. Maximum precision is achieved when $\lambda=x_{T}$ [7] and $x_{0}=3 x_{T}$. For the nonlinear decay model we verified numerically the existence of such an optimal decay rate; see Fig. 1(d). In general, in the experimentally relevant range $x_{T} \sim L / 2, x_{0} \sim \lambda$, we find $\omega_{s-s}$ $<\omega_{\text {lin }}<\omega_{\text {quad }}$. Although both the morphogen density and slope affect $\omega$, the value of the slope is the more important: the source-sink model has the steepest, and the quadratic model the least steep, profile, thereby generating the above ordering. Comparing this inequality with our earlier result $W_{\text {quad }}<W_{\text {lin }}<W_{s-s}$, the ordering is reversed, so that, for example, the quadratic decay model performs best on external fluctuations but worst on internal fluctuations.

The robustness of our three models to the combined effects of internal and external fluctuations can now be com- 

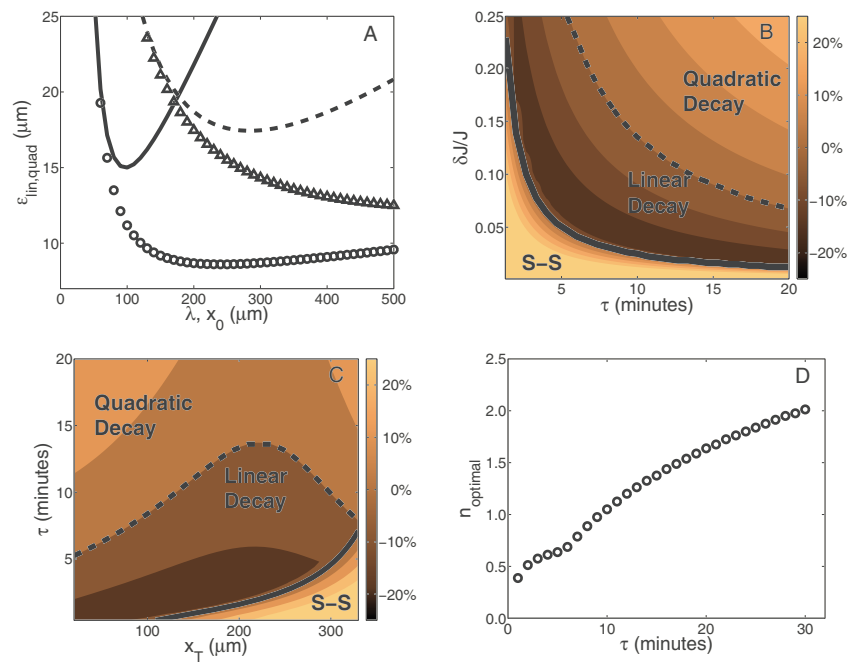

FIG. 2. (Color online) Optimizing morphogen profiles. (a) $\epsilon_{\text {lin }}$ versus $\lambda$ (solid line) and $\epsilon_{\text {quad }}$ versus $x_{0}$ (dashed line) with $\omega_{\text {lin }}(\bigcirc)$ and $\omega_{\text {quad }}(\triangle)$. The contributions solely from external fluctuations, which are linear in $\lambda$ and $x_{0}$, respectively, are omitted for clarity. (b) Effective phase diagram for relative precision of the three models considered with varying internal and external fluctuations. (c) Relative precision of models with varying $x_{T}$ in presence of internal fluctuations with fixed $\delta J / J=0.1$. Solid and dashed lines in (b) and (c) correspond to $\epsilon_{\text {lin }}=\epsilon_{s-s}$ (solid line) and $\epsilon_{\text {lin }}=\epsilon_{\text {quad }}$ (dashed line) and color map defined in text. (d) Optimal $n$ for different averaging times $\tau$. Parameters unless stated otherwise: $J=2$ molecules $\mu \mathrm{m}^{-2} \mathrm{~s}^{-1}, \quad \delta J / J=0.1, \quad \Delta x=3 \times 10^{-3} \mu \mathrm{m}, \quad D=15 \mu \mathrm{m}^{2} \mathrm{~s}^{-1}, \quad D_{0}$ $=0.5 \mu \mathrm{m}^{2} \mathrm{~s}^{-1}, \quad x_{T}=240 \mu \mathrm{m}, \tau=8 \mathrm{~min}, N_{\text {spat }}=0.06 \tau$, and $L$ $=550 \mu \mathrm{m}$.

pared. Since internal and external fluctuations are statistically independent, the total width is given by $\epsilon=\sqrt{\omega^{2}+W^{2}}$. We find that the minimum in $\epsilon_{\text {lin }}$ with respect to $\lambda$ (and in $\epsilon_{\text {quad }}$ with respect to $x_{0}$ ) becomes more pronounced than is the case with internal noise alone [see Fig. 2(a)]. As a result, the penalty in terms of reduced precision when using nonoptimized parameters is now more severe than in the internal noise-only case. Note that the optimal values of $\lambda$ and $x_{0}$ are reduced compared to their values when considering internal noise alone; see Fig. 2(a). For our chosen parameters, the optimal linear decay model is $15 \%$ more precise than the optimal quadratic decay model, a significant difference given that expression boundaries can be precise to around 1-2\% of embryo length $[8,14]$.

\section{OPTIMIZING MORPHOGEN PROFILE SHAPE}

We investigate which model gives the most precise positional information when subjected to embryo-to-embryo fluctuations (parametrized by $\delta J / J$ ) and internal fluctuations (parametrized by the averaging time $\tau$ ). For each $\delta J / J$ and $\tau$, we compute the optimal decay rates for the linear and quadratic decay models as above. We then build up an effective phase diagram in the $\tau-\delta J / J$ plane determining the most precise morphogen profile for particular levels of external and internal noise; see Fig. 2(b). The parameter values used are similar to those found from experiments on $h b$ expression in the Drosophila embryo [8,13,14,28]. $J$ is kept constant between the models representing fixed resource expenditure. The color map is defined as $\left(\epsilon_{\text {lin }}-\epsilon_{a}\right) / \epsilon_{\text {lin }} \times 100 \%$ where $\epsilon_{a}$ is the smaller of $\epsilon_{\text {quad }}$ and $\epsilon_{s-s}$. The percentage change is negative when the linear decay profile is most precise. For small times or small $\delta J / J$, profiles from the sink-source model are preferred as this model is best able to buffer the dominant internal fluctuations. For large $\delta J / J$ or large $\tau$ quadratic decay profiles are selected, as now the quadratic model is now able to best buffer the dominant external fluctuations. At intermediate values of $\delta J / J$ and $\tau$, when $W$ and $\omega$ are similar, exponential profiles are preferred as they provide the best compromise between robustness to both internal and external noise. From such diagrams we can build up a qualitative understanding of why particular morphogen profiles are selected depending upon the dominant sources of noise. We emphasize that our methodology is general and not dependent on the specific parameter values used above. For example, if $D=D_{0}$ then quadratic decay would be favored in a wider region of the phase space, but there would still be conditions when linear decay, or the source-sink model, would deliver higher precision.

\section{MULTIPLE TARGETS}

In many developmental systems morphogens are directly involved in the expression of several target genes at different positions throughout the system (e.g., orthodenticle ( $x_{T}$ $\approx 125 \mu \mathrm{m})[29]$ and $h b\left(x_{T} \approx 240 \mu \mathrm{m}\right)$ are both targets of $\mathrm{Bcd}$ ). We consider optimizing the morphogen profiles at one expression boundary and then compare how precisely the profiles determine a second boundary at a different position (keeping $\delta J / J$ fixed). Using the same optimized morphogen profiles as above we find that exponential profiles, unlike the other gradients, lead to the best precision at both short and long distances for short times $(\tau<10 \mathrm{~min})$; see Fig. 2(c). This flexibility is a potential explanation for the widespread use of exponential profiles in developmental systems.

\section{GENERALIZED MORPHOGEN PROFILES}

To demonstrate the generality of our results, we now solve Eq. (1) for a general $n>0$ and calculate the corresponding widths, $\epsilon_{n}$. We impose the constraint $\rho(x)>0$ everywhere across the embryo since morphogen gradients must typically specify multiple threshold positions (see above). Optimal degradation rates are found to be a general feature of such profiles. As demonstrated in Fig. 2(d), we find an optimal $n$ for a given averaging time, i.e., an optimal profile shape. Extending these ideas to the results shown in Fig. 2(b), for a fixed $\delta J / J$ we find a qualitatively similar picture with small $n$ favored at short averaging times, close to exponential profiles at intermediate times, and larger $n$ at longer times. Going beyond a simple reaction-diffusion model, Ref. [2] has shown, independent of the specific form of degradation, morphogen gradients robust to external source fluctuations must have rapid morphogen decay close to the source but, necessarily, significantly slower decay at large distances. Such profiles will be sensitive to internal noise due to the 
presence of flatter gradients away from the source. We see that, even for generalized degradation, profiles robust to external noise (e.g., algebraic profiles) will be poor at buffering internal noise, thereby further demonstrating the generality of our conclusions.

Intriguingly, the best characterized morphogens Bcd, Dpp, and Wingless in Drosophila all have exponential profiles and their decay lengths are significantly less than their respective values of $x_{T}[14,15]$. This is consistent with our conclusion that decay lengths adapt to buffer the combined effects of internal and external fluctuations and that (close to) exponential profiles perform well when buffering combined internalexternal fluctuations for a range of $x_{T}$ (since all three morphogens have multiple target genes). Experimentally, internal and external fluctuations can be distinguished [8]. Our work, therefore, makes clear and testable predictions: if external noise dominates, we predict that algebraic profiles will be favored; if internal and external fluctuations are of a similar magnitude then (close to) exponential profiles will be preferred; and if internal noise is the dominant source of error we expect (close to) linear morphogen profiles. Clearly, more complex processes may also be involved in the formation of these gradients, including, for example, transcytosis, nuclear trapping, pre-steady-state measurement or mRNA gradients $[3,12,30,31]$. In some circumstances, morphogen systems are able to scale with embryo length $[8,14,32]$. Nevertheless, once these additional effects are better characterized, our concepts can still be readily applied. In conclusion, attaining maximal robustness to the combined effects of internal and external noise may be a powerful unifying principle in understanding the fundamental design of morphogen systems.

\section{ACKNOWLEDGMENTS}

We thank Enrico Coen and Richard Morris for insightful comments. M.H. thanks The Royal Society for funding.
[1] A. D. Lander, Cell 128, 245 (2007).

[2] A. Eldar et al., Dev. Cell 5, 635 (2003).

[3] T. Bollenbach, K. Kruse, P. Pantazis, M. Gonzalez-Gaitan, and F. Julicher, Phys. Rev. Lett. 94, 018103 (2005).

[4] D. M. Umulis, M. B. O'Connor, and H. G. Othmer, Curr. Top. Dev. Biol. 81, 65 (2008).

[5] H. C. Berg and E. M. Purcell, Biophys. J. 20, 193 (1977).

[6] W. Bialek and S. Setayeshgar, Proc. Natl. Acad. Sci. U.S.A. 102, 10040 (2005).

[7] F. Tostevin, P. R. ten Wolde, and M. Howard, PLOS Comput. Biol. 3, e78 (2007).

[8] T. Gregor et al., Cell 130, 153 (2007).

[9] J. L. England and J. Cardy, Phys. Rev. Lett. 94, 078101 (2005).

[10] Manu et al., PLoS Biol. 7, e1000049 (2009).

[11] O. Crauk and N. Dostatni, Curr. Biol. 15, 1888 (2005).

[12] S. Bergmann et al., PLoS Biol. 5, e46 (2007).

[13] T. Gregor et al., Cell 130, 141 (2007).

[14] B. Houchmandzadeh, E. Wieschaus, and S. Leibler, Nature (London) 415, 798 (2002).

[15] A. Kicheva et al., Science 315, 521 (2007).

[16] G. Tkačik, C. G. Callan, Jr., and W. Bialek, Proc. Natl. Acad. Sci. U.S.A. 105, 12265 (2008).

[17] E. Emberly, Phys. Rev. E 77, 041903 (2008).
[18] F. Crick, Nature (London) 225, 420 (1970).

[19] D. Ben-Zvi et al., Nature (London) 453, 1205 (2008).

[20] Y. Sakai et al., Genes Dev. 15, 213 (2001).

[21] Y. F. Wu, E. Myasnikova, and J. Reinitz, BMC Syst. Biol. 1, 52 (2007).

[22] D. Lepzelter and J. Wang, Phys. Rev. E 77, 041917 (2008).

[23] H. H. McAdams and A. Arkin, Proc. Natl. Acad. Sci. U.S.A. 94, 814 (1997).

[24] G. Tkačik and W. Bialek, Phys. Rev. E 79, 051901 (2009).

[25] U. C. Täuber, M. Howard, and B. P. Vollmayr-Lee, J. Phys. A 38, R79 (2005).

[26] G. Tkačik, T. Gregor, and W. Bialek, PLoS ONE 3, e2774 (2008).

[27] T. Erdmann, M. Howard, and P. R. ten Wolde, e-print arXiv:0907.4289.

[28] T. Gregor et al., Proc. Natl. Acad. Sci. U.S.A. 102, 18403 (2005).

[29] R. Finkelstein and N. Perrimon, Nature (London) 346, 485 (1990).

[30] M. Coppey, A. M. Berezhkovskii, Y. Kim, A. N. Boettiger, and S. Y. Shvartsman, Dev. Biol. 312, 623 (2007).

[31] A. Spirov et al., Development 136, 605 (2009).

[32] F. He et al., Dev. Cell 15, 558 (2008). 